\title{
Past, present, and future of hormonal therapy in recurrent endometrial cancer
}

\author{
This article was published in the following Dove Press journal: \\ International Journal of Women's Health \\ 2 May 2014 \\ Number of times this article has been viewed
}

\author{
Matthew J Carlson \\ Kristina W Thiel \\ Kimberly K Leslie \\ Department of Obstetrics and \\ Gynecology, University of lowa, \\ lowa City, IA, USA
}

Correspondence: Kimberly K Leslie Department of Obstetrics and Gynecology, University of lowa, 200 Hawkins Drive, lowa City, IA 52242, USA

Tel +I 3193561976

Email kimberly-leslie@uiowa.edu

\begin{abstract}
Endometrial cancer is a heterogeneous disease. Type I cancers are hormonally driven, typically present with a low grade at an early stage, and are of endometrioid histology. These cancers are often cured by surgery, and the rate of recurrence is low. Type II cancers are less differentiated, often appear at a later stage, and are of serous, clear cell, or high grade endometrioid histology. The risk of recurrence in these cancers is much higher than with type I tumors. Isolated pelvic recurrences can be treated with radiation or exenteration, but systemic disease is fatal. It is in these recurrent patients, where prolongation of progression-free survival is the goal, that hormonal therapy can have the greatest benefit. In selected patients, hormonal therapy can be as effective as cytotoxic chemotherapy, without the toxicity and at a much lower cost. Here we review the evidence for treatment of patients suffering from recurrent endometrial cancer with hormonal therapy and explore avenues for the future of hormonal treatment of endometrial cancer. Currently, progesterone is the hormonal treatment of choice in these patients. Other drugs are also used, including selective estrogen receptor modulators, aromatase inhibitors, and gonadotropin-releasing hormone antagonists. Hormonal treatment of recurrent endometrial cancer relies on expression of the hormone receptors, which act as nuclear transcription factors. Tumors that express these receptors are the most sensitive to therapy; it is for this reason that patient selection is vitally important to the successful treatment of recurrent endometrial cancer with hormonal therapy.
\end{abstract}

Keywords: hormonal therapy, recurrent endometrial cancer

\section{Introduction}

Endometrial cancer is often diagnosed at an early stage, due in large part to the symptomatic nature of the disease which presents with uterine/vaginal bleeding. Data from the National Cancer Institute's Surveillance, Epidemiology, and End Results program demonstrated that $73 \%$ of endometrial cancer patients have stage I disease at diagnosis, whereas approximately $10 \%$ are diagnosed with stage II disease. ${ }^{1,2}$ The 5-year survival for stage I patients is $85 \%-91 \%$. $^{1,2}$ Most patients are treated surgically and, based on specific pathologic and patient criteria (age, grade of tumor, depth of invasion, presence of lymphovascular space invasion), the patient may be treated with radiation therapy after surgery. Regardless, the recurrence rate in stage I patients is low, but recurrence is not completely absent. In the Gynecologic Oncology Group (GOG) LAP2 study, where patients were randomized to surgery by conventional open laparotomy versus laparoscopy, the recurrence rates at 3 years were approximately $10 \%$ in each arm for patients with stage I-II endometrial cancer. ${ }^{3}$ 
Advanced stage (stage III-IV) endometrial cancer is less common, and, at the time of surgery, is frequently associated with metastases to the ovaries, abdomen, or lymph nodes. Occasionally, the disease is found outside the abdomen. Patients with advanced endometrial cancer are usually treated with surgical debulking followed by radiation, chemotherapy, or a combination thereof. The 5-year survival in these patients is $30 \%-40 \%$ and $60 \%-70 \%$ for para-aortic and pelvic nodal involvement, respectively. ${ }^{2}$ Based on these statistics, it is clear that recurrence is common. For example, in the recent interim analysis of the GOG 209 protocol, which randomized patients with advanced endometrial cancer to chemotherapy with paclitaxel, doxorubicin, and cisplatin versus carboplatin and paclitaxel, the median progression-free survival was 14 months in both arms, and overall survival was 32 and 38 months, respectively. ${ }^{4}$

In general, recurrent endometrial cancer is treatable but not curable unless it is confined to the vaginal cuff or pelvis. Widely metastatic recurrence is fatal. The treatment for recurrent endometrial cancer depends on the anatomic location of the recurrence. If the recurrence is confined to the pelvis, and the patient has not received whole pelvic radiation therapy, radiotherapy is the treatment of choice. These patients experience a 5-year local control rate of $42 \%-65 \%$ and a 5 -year overall survival rate of $31 \%-53 \% .^{5-7}$ While this treatment approach has a good response rate, it is not without side effects. Indeed, the rate of grade 4 complications has been reported to be as high as $9 \%$, and many patients who receive radiation to the pelvis experience vaginal stenosis, cystitis, proctitis, and chronic diarrhea, which significantly impacts their life..$^{5-7}$ In the case of systemic metastases, chemotherapy has a poor track record in improving survival, with most trials reporting response rates of less than $20 \%$, progression-free survival of 3-6 months, and overall survival of less than 12 months when using chemotherapy in the recurrent setting. 8,9

Given that patients with advanced and recurrent disease experience suboptimal response rates and frequent life-altering side effects, continuing cytotoxic chemotherapy when the likelihood of response is only $20 \%$ is problematic. This is particularly true when other agents are available that have fewer side effects and are as or more effective in a selected population of patients. The side effects of hormonal therapy depend on the agents used, but are generally mild and do not include grade 3 or 4 toxicities. Progesterone, referred to as the "ultimate endometrial tumor suppressor," has been used for many years in the treatment of endometrial cancer. ${ }^{10}$ In order to highlight the options which should be considered in the treatment of women with advanced endometrial cancer, this review focuses on hormonal treatment. Hormonal therapy for endometrial cancer falls into two broad categories, ie, progestin-containing regimens and antiestrogen regimens. The most commonly used hormonal agents aimed at reducing estrogen signaling include selective estrogen receptor modulators, gonadotropin-releasing hormone antagonists, and aromatase inhibitors.

\section{Progestins}

Progesterone and its chemical derivatives, referred to as progestins, have been widely used in the treatment of endometrial cancer because these agents induce differentiation of the glandular endometrial epithelium. The side effects of progestins include a possible increased risk of thromboembolic events (although less risky than estrogens with respect to thromboembolism), peripheral edema, and increased appetite. Hormonal therapy with progestins was first studied by the GOG in the 1980s, but had been used in clinical practice for many years prior to that. ${ }^{11}$ The results of GOG 48 were presented in 1986 and showed a 17\% response rate among 331 patients with advanced or recurrent endometrial cancer. ${ }^{12}$ These patients were given medroxyprogesterone acetate $50 \mathrm{mg}$ three times daily until disease progression or toxicity precluded further treatment. The $17 \%$ response rate seen was less than previously reported, but the population studied was more homogeneous than in previous studies. The GOG later published protocol 81, in which 299 women with advanced or recurrent endometrial carcinoma were randomized to two different doses of oral medroxyprogesterone acetate $(200 \mathrm{mg} /$ day or $1,000 \mathrm{mg} /$ day) until unacceptable toxicity intervened or their disease progressed. Among 145 patients who received the low-dose regimen, there were 25 complete (17\%) and eleven partial $(8 \%)$ responses for an overall response rate of $25 \%$. More medroxyprogesterone acetate was not better; the patients who received the high-dose regimen experienced $14(9 \%)$ complete and ten $(6 \%)$ partial responses for an overall response rate of $15 \%$. Moreover, median progression-free survival was 3.2 and 2.5 months and median overall survival 11.1 and 7.0 months for the low-dose and high-dose regimens, respectively. ${ }^{12}$

Toward understanding why responses in these two GOG protocols were less than anticipated based on historical observations, investigations of the estrogen receptor (ER) and progesterone receptor (PR) were undertaken. Although receptor levels were not assessed at a central reference laboratory, pretreatment levels, determined by the entering institutions, were available for 132 of the 299 eligible patients. 
Patients were considered receptor-positive if they had more than $10 \mathrm{fmoles} / \mathrm{mg}$ of ER and 50 moles/mg of PR. Not surprisingly, there was a positive correlation between response and receptor status. The response rate was $8 \%$ (seven of 86 patients) for patients who were PR-negative and 37\% (17 of 46) for patients who were PR-positive $(P<0.001)$. Similar findings were observed for ER ( $7 \%$ for ER-negative versus $26 \%$ for ER-positive; $P<0.005) .{ }^{12}$ Based on these findings, a subsequent GOG protocol, GOG 119, examined the effect of tamoxifen with intermittent medroxyprogesterone acetate. The hypothesis was that tamoxifen, acting through ER, would increase expression of PR and thus sensitivity to medroxyprogesterone acetate. In this trial, 61 patients with advanced or recurrent endometrial cancer received $20 \mathrm{mg}$ of tamoxifen twice daily and alternating weeks of medroxyprogesterone acetate at $200 \mathrm{mg}$ daily. Medroxyprogesterone acetate was given intermittently because it was thought that sustained activation of PR might lead to receptor degradation, thus minimizing the differentiating effect of progestin. This study reported a $33 \%$ overall response rate with a progression-free survival of 3 months and an overall survival of 13 months. ${ }^{13}$ Table 1 provides a synopsis of studies of hormonal treatment of recurrent endometrial cancer.

Further analysis of the relationship between hormone receptor expression and response revealed a strong correlation between ER expression and response to treatment. Indeed, the single best predictor of response was ER, which was significantly associated with clinical response

Table I Clinical trials of hormonal therapy in recurrent endometrial cancer

\begin{tabular}{|c|c|c|c|c|c|}
\hline Reference & (n) & Treatment & $\mathbf{R R}$ & PFS & OS \\
\hline Thigpen et al & 154 & MPA I,000 mg daily versus & 15 & 2.5 & 7 \\
\hline$(G O G 8 I)^{12}$ & 145 & MPA 200 mg daily & & & \\
\hline $\begin{array}{l}\text { Lentz et al } \\
(\text { GOG } 12 \mid)^{39}\end{array}$ & 58 & MA 800 mg daily & 25 & 3.2 & 11.1 \\
\hline $\begin{array}{l}\text { Thigpen et al } \\
(\text { GOG } 8 I-F)^{20}\end{array}$ & 68 & Tamoxifen 20 mg BID & 10 & 1.9 & 8.8 \\
\hline $\begin{array}{l}\text { Whitney et al } \\
(\text { GOG II9) }\end{array}$ & 58 & $\begin{array}{l}\text { MPA } 100 \mathrm{mg} \text { BID on } \\
\text { alternating weeks plus } \\
\text { tamoxifen } 20 \mathrm{mg} \text { daily } \\
\text { continuous }\end{array}$ & 33 & 3 & 13 \\
\hline $\begin{array}{l}\text { Fiorica et al } \\
(\text { GOG I53) }\end{array}$ & 56 & $\begin{array}{l}\text { MA } 80 \mathrm{mg} \text { BID } \times 3 \text { weeks } \\
\text { alternating with tamoxifen } \\
20 \mathrm{mg} \text { BID } \times 3 \text { weeks }\end{array}$ & 27 & 2.7 & 14 \\
\hline $\begin{array}{l}\text { Rose et al } \\
(\text { GOG I68) }\end{array}$ & 23 & Anastrozole I mg daily & 9 & I & 6 \\
\hline Ma et $\mathrm{a}^{29}$ & 32 & Letrozole $2.5 \mathrm{mg}$ daily & 9.4 & 3.9 & 8.8 \\
\hline Asbury et al ${ }^{24}$ & 40 & $\begin{array}{l}\text { Goserelin acetate } 3.6 \mathrm{mg} \\
\text { monthly }\end{array}$ & 11 & 1.9 & 7.3 \\
\hline
\end{tabular}

Abbreviations: MPA, medroxyprogesterone acetate; MA, megestrol acetate; PFS, progression-free survival; OS, overall survival; RR, response rate; GOG, Gynecologic Oncology Group; BID, twice daily. and survival. Progesterone receptors were investigated according to their isoforms, PRA and PRB. Both forms arise from alternative promoters on the same gene and can form homo $(\mathrm{A} / \mathrm{A}, \mathrm{B} / \mathrm{B})$ or hetero $(\mathrm{A} / \mathrm{B})$ dimeric units. The isoforms are identical except that $\mathrm{PRB}$ has a longer N-terminus consisting of 164 amino acids not present in PRA. ${ }^{14}$ While there was no significant correlation between PR expression and response, likely due to the small number of patients, there was a strong correlation between ER and PRA but not PRB expression. This study was unique in that it required a pretreatment biopsy in order to determine a correlation between hormone receptor expression and clinical response to the regimen. This study also established a method to quantitate receptor expression levels, termed the HSCORE, which takes into account the percentage of receptor-positive cells and the staining intensity. Importantly, the median survival of patients with an ER HSCORE >75 was 19 months, which is comparable with the 15-18-month median survival time reported with doxorubicin, cisplatin, and paclitaxel with granulocyte colony-stimulating factor support and paclitaxel and cisplatin with granulocyte colony-stimulating factor support. ${ }^{15}$ These findings underscore the potential effectiveness of hormonal therapy if patients are chosen based upon positive receptor status.

Overall, progestins remain a valid option not only for patients with recurrent receptor-positive tumors after chemotherapy, but also for patients with well differentiated (low grade) endometrioid adenocarcinomas that are positive for hormone receptors but are not suitable for chemotherapy. In a recent systematic review, $11 \%-56 \%$ of grade 1 and 2 tumors were shown to respond to progestins, with the response rate generally higher for PR-positive tumors. Importantly, toxicity was remarkably low, with the rate of grade 3 and 4 events being less than $5 \% .{ }^{16}$ However, it remains unclear why many hormone receptor-positive tumors fail to respond to progestins, while a small percentage of ER/PR-negative tumors respond to progestin therapy. Interestingly, some patients treated with progestins have achieved long-term responses exceeding 2 years. ${ }^{17}$ As discussed below, it is possible that ER and PR levels can be induced with new hormonal regimens in combination with targeted agents in order to enhance long-term responses $;{ }^{18}$ however, these multiagent hormonal regimens are not yet in clinical trials.

\section{Agents aimed at reducing estrogen signaling}

It is understood that excessive or unopposed estrogen in the endometrium leads to hyperplasia and ultimately cancer. 
It is equally well known that estrogen stimulates growth within endometrial tissues, and this has been proven in clinical trials and with the observed significant increase in endometrial cancer, which spiked during the era of unopposed estrogen therapy for hormonal replacement. ${ }^{19}$ These data have motivated investigation of several other agents, including selective estrogen receptor modulators (SERMs), gonadotropin-releasing hormone $(\mathrm{GnRH})$ analogs, and aromatase inhibitors. All of these agents function to either decrease the overall levels of estrogen in the body or to block its ability to interact with the ER.

\section{Selective estrogen receptor modulators}

SERMs are competitive estrogen inhibitors that bind to the ER. Agents in this class include tamoxifen, raloxifene, and arzoxifene, the side effects of which include transient thrombocytopenia and hot flashes. Tamoxifen has been examined in several trials in recurrent endometrial cancer. As a single agent, $20 \mathrm{mg}$ of tamoxifen was given twice daily to 68 patients with recurrent endometrial carcinoma until toxicity was unacceptable or disease progressed. Unfortunately, tamoxifen demonstrated limited activity as a single agent, with an overall response rate of only $10 \%$, and did not warrant further study, according to the GOG. ${ }^{20}$ Raloxifene yielded similarly disappointing results. ${ }^{21}$ However, two Phase II studies of arzoxifene for recurrent endometrial cancer demonstrated significant activity at $20 \mathrm{mg}$ /day in patients with metastatic or recurrent endometrial cancer. ${ }^{22,23}$ Specifically, observed clinical response rates were $25 \%$ and $31 \%$, with median response durations of 19.3 and 13.9 months, respectively. Toxicity was mild, except for two cases of pulmonary embolism that might have been drug-related. ${ }^{22}$ Further investigation is warranted to verify these preliminary response rates and the clinical significance of the stable disease cases, to compare clinical outcomes with those in progestin-treated women, and to elucidate the mechanisms of action of SERMs in this disease.

\section{Gonadotropin-releasing hormone analogs}

GnRH analogs downregulate follicle-stimulating hormone and leutinizing hormone receptors in the pituitary, with a subsequent fall in gonadotropin levels. The loss of systemic gonadotropin leads to a decrease in estrogen levels, which can lead to hot flashes. GnRH analogs have proven efficacy in the treatment of prostate cancer, where they have become standard therapy because of their improved therapeutic ratio when compared with estrogens or orchiectomy. These analogs promote an initial increase in pituitary gonadotropins, followed by a profound suppression that results in a decrease of gonadal sex hormones to castrate levels. Initial studies of goserelin acetate, a GnRH analog, at a dose of $3.6 \mathrm{mg}$ per month in endometrial cancer suggested activity. In a study of 40 patients performed by the GOG, an $11 \%$ response rate was observed. Interestingly, one patient who demonstrated a complete response had previously responded and subsequently progressed while receiving alternating progestin and tamoxifen therapy. There appeared to be a relationship between the grade and durability of response, with two of eight patients with grade I disease achieving a response, and three of 14 patients with grade II tumors achieving a response. No patients with grade III tumors responded to therapy. However, the overall response rate was suboptimal at $11 \% .{ }^{24}$ Because of the poor responses to GnRH analogs, they are not generally used for recurrent endometrial cancer. However, the complete and partial responses in a subset of patients suggest that some patients may respond to $\mathrm{GnRH}$ analogs, although no translational investigations for predictive biomarkers have been reported.

\section{Aromatase inhibitors}

Aromatase is the key enzyme that catalyzes conversion of androgens to estrogens in postmenopausal women. Aromatase inhibitors are nonsteroidal competitive inhibitors of the aromatase enzyme that act to decrease systemic and intratumoral estrogen levels. Several studies have shown increased aromatase expression within endometrial tumor tissue relative to surrounding normal tissue. ${ }^{25-27}$ The side effects include hot flashes and osteoporosis with prolonged use.

Aromatase inhibitors have largely failed in endometrial cancer. Rose et al showed in 23 patients that anastrozole (1 $\mathrm{mg}$ a day for 28 days) has minimal activity, with only a $9 \%$ partial response rate and a progression-free interval of 1-6 months. ${ }^{28}$ The National Cancer Institute of Canada Clinical Trial Group reported a Phase II study of letrozole in patients with advanced or metastatic endometrial cancer who may have received progestins and at most one prior line of chemotherapy in the metastatic setting. One complete response and two partial responses were noted, and eleven patients had stable disease for a median duration of 6.7 months. The median overall survival was 8.8 months and the median time to progression was 3.9 months. The expression of various biomarkers relevant for endometrial cancer failed to correlate with response. The most common letrozolerelated toxicities included grade 1 and 2 hot flashes $(28 \%)$, followed by fatigue (12.5\%) and anemia. ${ }^{29}$ 
In summary, hormonal regimens aimed at reducing estrogen signaling without adding a progestin have not shown significant promise to date. However, the complete and partial responses of a small number of patients who received SERMs, GnRH analogs, or aromatase inhibitors do not rule out the possibility for activity in a selected group of patients. One study of letrozole in hormone receptor-positive advanced or metastatic endometrial cancer (NCT00171808) and one study in ER-positive advanced or metastatic endometrial cancer (NCT00333086) have finished enrollment, but no results are currently published or available for review. If responses occurred, there is the potential to study these subjects to better understand which cases are most likely to respond to antiestrogen hormonal therapy. Further development of these strategies will depend upon the results of these trials and the future potential of creating combinations of agents aimed at reducing estrogen signaling, while at the same time inducing cellular differentiation (as with the addition of a progestin, for example).

\section{Importance of patient selection}

A question that clinicians must answer before considering a patient as a candidate for hormonal therapy is whether the recurrent tumor still expresses the hormone receptors needed for successful treatment. There are precedents in the literature indicating that progression of endometrial cancer is associated with loss of hormone receptors. For example, investigators at Memorial Sloan Kettering Cancer Center examined 34 cases of endometrial cancer in which primary and recurrent tumors were available. ${ }^{30}$ In addition to histologic classification, primary and matched recurrent tumors were analyzed for expression of PR as well as other markers of interest, such as p53, p16, and DNA mismatch repair proteins (MLH1, MSH2/6, and PMS2). Compared with endometrioid carcinoma patients, serous carcinoma patients were older, presented at a higher stage, and had shorter survival. Serous carcinomas were the most common recurrent endometrial carcinoma, and $62 \%$ displayed similar morphology when comparing primary and recurrent carcinomas. Seven of 13 endometrioid carcinomas (54\%) had morphologically discordant recurrence. Serous and morphologically ambiguous carcinomas demonstrated relative morphologic fidelity compared with endometrioid carcinomas.

Seven of 23 matched pairs displayed discordant PR results, with five cases, including both endometrioid and serous carcinomas, showing diminished PR expression at recurrence. These five cases comprised two serous, two grade 3, and one grade 2 tumors. Two of the seven cases with discordant PR showed increased PR expression, and both were of serous histology at initial diagnosis and on recurrence. All seven of these cases had histology that was concordant between the primary and recurrent tumors. Interestingly, PR expression was the same in all of the tumors with discordant histology. Seven of these 13 cases were either grade 1 or 2 endometrioid tumors with moderate to high PR expression. The difficulty in studying this disease should be noted; in this study, between 2000 and 2010, there were only 34 cases that had matched primary and recurrent tumor specimens available. The investigators were limited in the staining that could be accomplished using the residual tumor blocks. Further analysis of ER expression in this study, as well as performing confirmatory investigations in a larger cohort of patients, might be enlightening, but some limited conclusions can be drawn from this report. ${ }^{30}$ The data suggest that when treating recurrent serous carcinoma of the endometrium, it is reasonable to make decisions regarding therapy on the primary tumor. However, for recurrent endometrioid carcinoma, where the loss of PR appears to be common, sampling the recurrent tumor to test for hormone receptor expression is worthwhile if hormonal therapy is planned in the future.

In general, current best practice dictates that patients must be well selected for treatment with hormonal agents, given that the patients most likely to respond will be those with ongoing ER and PR expression. However, a future opportunity is on the horizon to address even those tumors without ER and PR. The ideal next step in the treatment of hormone receptor-negative tumors is to identify combinatorial regimens that promote re-expression of hormone receptors. Potential strategies are discussed below which could significantly broaden the applicability of hormonal therapy to many more patients with endometrial cancer.

\section{Current work and future directions Onapristone}

Onapristone is a PR antagonist that has demonstrated antitumor activity in preclinical and clinical studies of hormonedependent tumors. ${ }^{31,32}$ Its mechanism of action is thought to be a direct result of binding to the PR and preventing it from binding to DNA, thereby substantially reducing or eliminating PR-induced transcription, resulting in death or differentiation of the malignant cells..$^{31}$ Onapristone is currently under investigation in patients with PR-expressing tumors (NCT02052128). 


\section{Endoxifen}

Endoxifen is one of the active metabolites of tamoxifen. Much work has been done in recent years showing that slow metabolizers, ie, those with polymorphisms in cytochrome P450 enzymes, have poorer breast cancer outcomes when treated with tamoxifen. ${ }^{33}$ This has sparked new interest in utilizing endoxifen as an agent on its own and it is currently being investigated in hormone receptor-positive solid tumors (NCT01273168).

\section{Histone deacetylase inhibitors}

Several groups have explored the mechanisms underlying loss of hormone receptor expression in endometrial cancer, with the goal of identifying new therapeutic targets that can restore functional hormone receptor expression and thereby sensitivity to hormonal therapy. Work by our group and others identified epigenetic silencing of PR as one such mechanism by which the cancer cells repress expression of PR. ${ }^{11,18,34-36}$ Moreover, we found that treatment of endometrial cancer cells in vitro with a histone deacetylase inhibitor induced upregulation of PR messenger (m)RNA. ${ }^{18}$ This led to restored PR protein expression within 24 hours and sustained expression for 72 hours. PR expression persisted even in the presence of progesterone, and the re-expressed receptor was found to be functional, as evidenced by localization to the nucleus and arrest in G1. Thus, epigenetic modulation is one strategy to restore functional PR expression and shows promise for future hormonal treatment of endometrial cancer.

Another approach is to harness the technology of gene therapy. In proof-of-concept studies using a xenograft model of endometrial cancer, adenoviral-mediated expression of PR sensitized tumors to progestin therapy. ${ }^{37}$

\section{Conclusion}

Recurrent endometrial cancer is a complicated disease with a poor prognosis. In general, it can be treated with many modalities, including radiation therapy, chemotherapy, and surgery. None of these treatments are without significant side effects, and caution should be exercised when making therapeutic decisions. Hormonal therapy offers a tolerable and effective treatment for recurrent endometrial cancer. Patients should be carefully selected based on hormone receptor expression. Indeed, recent work using the Cancer Genome Atlas data set has challenged the traditional classification of endometrial cancers into "type I" and "type II", by reclassifying tumors into multiplatform subtypes based on mRNA expression, somatic copy number alterations, microsatellite instability, and somatic nucleotide substitutions. ${ }^{38}$ Tumors in the "low copy-number" cluster are typically hormone receptor-positive, whereas "high copy-number" tumors are devoid of ER and PR expression. While the future of cancer treatment is likely to lie in personalized medicine, the incorporation of genomic or proteomic profiling for individual patient tumors is still not routine in clinical practice. Selection of patients based on hormone receptor expression is a first step. In the future, with continued advances in epigenetic modulators and other agents, even tumors devoid of hormone receptors may be treated using molecularly enhanced hormonal therapy to boost hormone receptor expression.

\section{Disclosure}

The authors report no conflicts of interest in this work.

\section{References}

1. Trimble EL, Harlan LC, Clegg LX, Stevens JL. Pre-operative imaging, surgery and adjuvant therapy for women diagnosed with cancer of the corpus uteri in community practice in the United States. Gynecol Oncol. 2005;96(3):741-748

2. Creasman WT, Odicino F, Maisonneuve P, et al. Carcinoma of the corpus uteri. FIGO 26th Annual Report on the Results of Treatment in Gynecological Cancer. Int J Gynaecol Obstet. 2006;95 Suppl 1:S105-S143.

3. Walker JL, Piedmonte MR, Spirtos NM, et al. Laparoscopy compared with laparotomy for comprehensive surgical staging of uterine cancer: Gynecologic Oncology Group Study LAP2. J Clin Oncol. 2009; 27(32):5331-5336.

4. Miller D, Filiaci V, Fleming G, et al. Randomized phase III noninferiority trial of first line chemotherapy for metastatic or recurrent endometrial carcinoma: a Gynecologic Oncology Group study. Gynecol Oncol. 2012;125(3):771

5. Curran WJ Jr, Whittington R, Peters AJ, Fanning J. Vaginal recurrences of endometrial carcinoma: the prognostic value of staging by a primary vaginal carcinoma system. Int J Radiat Oncol Biol Phys. 1988;15(4): 803-808.

6. Sears JD, Greven KM, Hoen HM, Randall ME. Prognostic factors and treatment outcome for patients with locally recurrent endometrial cancer. Cancer. 1994;74(4):1303-1308.

7. Wylie J, Irwin C, Pintilie M, et al. Results of radical radiotherapy for recurrent endometrial cancer. Gynecol Oncol. 2000;77(1):66-72.

8. Levine DA, Hoskins WJ. Update in the management of endometrial cancer. Cancer J. 2002;8 Suppl 1:S31-S40.

9. Sonoda Y. Optimal therapy and management of endometrial cancer. Expert Rev Anticancer Ther. 2003;3(1):37-47.

10. Yang S, Thiel KW, De Geest K, Leslie KK. Endometrial cancer: reviving progesterone therapy in the molecular age. Discov Med. 2011;12(64): 205-212.

11. Yang S, Thiel KW, Leslie KK. Progesterone: the ultimate endometrial tumor suppressor. Trends Endocrinol Metab. 2011;22(4):145-152.

12. Thigpen JT, Brady MF, Alvarez RD, et al. Oral medroxyprogesterone acetate in the treatment of advanced or recurrent endometrial carcinoma: a dose-response study by the Gynecologic Oncology Group. J Clin Oncol. 1999;17(6):1736-1744.

13. Whitney CW, Brunetto VL, Zaino RJ, et al. Phase II study of medroxyprogesterone acetate plus tamoxifen in advanced endometrial carcinoma: a Gynecologic Oncology Group study. Gynecol Oncol. 2004;92(1):4-9.

14. Leslie KK, Sill MW, Fischer E, et al. A phase II evaluation of gefitinib in the treatment of persistent or recurrent endometrial cancer: a Gynecologic Oncology Group study. Gynecol Oncol. 2013;129(3): 486-494. 
15. Singh M, Zaino RJ, Filiaci VJ, Leslie KK. Relationship of estrogen and progesterone receptors to clinical outcome in metastatic endometrial carcinoma: a Gynecologic Oncology Group Study. Gynecol Oncol. 2007;106(2):325-333.

16. Decruze SB, Green JA. Hormone therapy in advanced and recurrent endometrial cancer: a systematic review. Int J Gynecol Cancer. 2007;17(5):964-978.

17. Markman M. Hormonal therapy of endometrial cancer. Eur J Cancer. 2005;41(5):673-675.

18. Yang S, Xiao X, Jia Y, et al. Epigenetic modification restores functional PR expression in endometrial cancer cells. Curr Pharm Des. July 19 , 2013. [Epub ahead of print.]

19. Allen NE, Tsilidis KK, Key TJ, et al. Menopausal hormone therapy and risk of endometrial carcinoma among postmenopausal women in the European Prospective Investigation Into Cancer and Nutrition. Am J Epidemiol. 2010;172(12):1394-1403.

20. Thigpen T, Brady MF, Homesley HD, Soper JT, Bell J. Tamoxifen in the treatment of advanced or recurrent endometrial carcinoma: a Gynecologic Oncology Group study. J Clin Oncol. 2001;19(2):364-367.

21. Elit L, Hirte H. Novel strategies for systemic treatment of endometrial cancer. Expert Opin Investig Drugs. 2000;9(12):2831-2853.

22. McMeekin DS, Gordon A, Fowler J, et al. A phase II trial of arzoxifene, a selective estrogen response modulator, in patients with recurrent or advanced endometrial cancer. Gynecol Oncol. 2003;90(1):64-69.

23. Burke TW, Walker CL. Arzoxifene as therapy for endometrial cancer. Gynecol Oncol. 2003 Aug;90(2 Pt 2):S40-S46.

24. Asbury RF, Brunetto VL, Lee RB, Reid G, Rocereto TF; Gynecologic Oncology Group. Goserelin acetate as treatment for recurrent endometrial carcinoma: a Gynecologic Oncology Group study. Am J Clin Oncol. 2002;25(6):557-560.

25. Che Q, Liu BY, Liao Y, et al. Activation of a positive feedback loop involving IL-6 and aromatase promotes intratumoral 17beta-estradiol biosynthesis in endometrial carcinoma microenvironment. Int J Cancer. December 18, 2013. [Epub ahead of print.]

26. Takahashi-Shiga N, Utsunomiya H, Miki Y, et al. Local biosynthesis of estrogen in human endometrial carcinoma through tumor-stromal cell interactions. Clin Cancer Res. 2009;15(19):6028-6034.

27. Segawa T, Shozu M, Murakami K, et al. Aromatase expression in stromal cells of endometrioid endometrial cancer correlates with poor survival. Clin Cancer Res. 2005;11(6):2188-2194.

28. Rose PG, Brunetto VL, VanLe L, Bell J, Walker JL, Lee RB. A phase II trial of anastrozole in advanced recurrent or persistent endometrial carcinoma: a Gynecologic Oncology Group study. Gynecol Oncol. 2000;78(2):212-216.
29. Ma BB, Oza A, Eisenhauer E, et al. The activity of letrozole in patients with advanced or recurrent endometrial cancer and correlation with biological markers - a study of the National Cancer Institute of Canada Clinical Trials Group. Int J Gynecol Cancer. 2004;14(4):650-658.

30. Soslow RA, Wethington SL, Cesari M, et al. Clinicopathologic analysis of matched primary and recurrent endometrial carcinoma. Am J Surg Pathol. 2012;36(12):1771-1781.

31. Robertson JFR, Willsher PC, Winterbottom L, Blamey RW, Thorpe S. Onapristone, a progesterone receptor antagonist, as first-line therapy in primary breast cancer. Eur J Cancer. 1999;35(2):214-218.

32. Schneider CC, Gibb RK, Taylor DD, Wan T, Gercel-Taylor C. Inhibition of endometrial cancer cell lines by mifepristone (RU 486). J Soc Gynecol Investig. 1998;5(6):334-338.

33. Jordan VC. New insights into the metabolism of tamoxifen and its role in the treatment and prevention of breast cancer. Steroids. 2007;72(13): $829-842$.

34. Sasaki M, Dharia A, Oh BR, Tanaka Y, Fujimoto S, Dahiya R. Progesterone receptor B gene inactivation and $\mathrm{CpG}$ hypermethylation in human uterine endometrial cancer. Cancer Res. 2001;61(1): 97-102.

35. Xiong Y, Dowdy SC, Gonzalez Bosquet J, et al. Epigenetic-mediated upregulation of progesterone receptor B gene in endometrial cancer cell lines. Gynecol Oncol. 2005;99(1):135-141.

36. Ren Y, Liu X, Ma D, Feng Y, Zhong N. Down-regulation of the progesterone receptor by the methylation of progesterone receptor gene in endometrial cancer cells. Cancer Genet Cytogenet. 2007;175(2): 107-116.

37. Dai D, Albitar L, Nguyen T, Laidler LL, Singh M, Leslie KK. A therapeutic model for advanced endometrial cancer: systemic progestin in combination with local adenoviral-mediated progesterone receptor expression. Mol Cancer Ther. 2005;4(1):169-175.

38. Cancer Genome Atlas Research Network; Kandoth C, Schultz N, Cherniack AD, et al. Integrated genomic characterization of endometrial carcinoma. Nature. 2013;497(7447):67-73.

39. Lentz SS, Brady MF, Major FJ, Reid GC, Soper JT. High-dose megestrol acetate in advanced or recurrent endometrial carcinoma: a Gynecologic Oncology Group Study. J Clin Oncol. 1996;14(2): 357-361.

40. Fiorica JV, Brunetto VL, Hanjani P, et al. Phase II trial of alternating courses of megestrol acetate and tamoxifen in advanced endometrial carcinoma: a Gynecologic Oncology Group study. Gynecol Oncol. 2004;92(1):10-14.
International Journal of Women's Health

\section{Publish your work in this journal}

The International Journal of Women's Health is an international, peerreviewed open-access journal publishing original research, reports, editorials, reviews and commentaries on all aspects of women's healthcare including gynecology, obstetrics, and breast cancer. The manuscript management system is completely online and includes

\section{Dovepress}

a very quick and fair peer-review system, which is all easy to use. Visit http://www.dovepress.com/testimonials.php to read real quotes from published authors. 